\title{
The Feasibility Research of GNSS Difference Positioning Based on Maritime Removed Datum
}

\author{
Jian Zhou, Yuyong Shang, Zhi Liu, Jun Yan \\ China Satellite Maritime Tracking and Control Department, Jiangyin, 214431, China
}

\begin{abstract}
In this paper, the use of mobile offshore base station to accurately determine the relative position of the target space and the use of methods, described in detail the relative positioning of the mobile base station technology is an important part of the project to carry out measurements at sea. By analyzing the current research, we analyze the development prospects of the technology, and preliminary experimental research program set up hardware and software environment, demonstrate the feasibility of the relative positioning of the mobile base station technology in offshore measurements.
\end{abstract}

Keywords: GNSS difference positioning; Removed datum; BDS

\section{Introduction}

A With the development of satellite navigation and positioning technology, GNSS positions technology in space applications in the deepening, making use of satellite navigation to a specific target dynamic differential positioning precision possible.

At sea, because there is no fixed reference station to provide a differential signal, in order to solve the positioning precision between two or more moving targets, we use dynamic differential satellite navigation technology, the base station is provided on the moving vehicle, in order to solve the mobile station accurate relative position with respect to the base station. In addition, the use of the base station sets the dynamic differential positioning system, but also help solve the strict maritime UAV formation flight, ship and marine navigation precise formations to enhance the measurement accuracy and other issues. In particular BDS to achieve global coverage in 2020, how to use the BD satellite navigation and positioning system for dynamic precision positioning technology has become a hot research.

\section{Analysis of Necessity}

As we all know, in the past, marine surveying, the GNSS differential positioning need to use one or more fixed reference stations via the radio base station transmits data to the rover, this model can meet the needs of most applications, but in some cases, due to the limited coverage of differential positioning data sent by a fixed reference station can not meet the formation flying, remote measurement applications that require dynamic specific objectives. In the conventional differential position, the position of fixed reference stations is precisely known in advance, the prerequisites dynamic GNSS carrier phase technology is not, and thus the basic theory of traditional differential positioning difficult in the dynamic differential reference station positioning applications.

In order to solve between two or more moving targets with high precision relative positioning of the problem, it will use GNSS carrier relative positioning dynamic measurement technology, the base station is provided on a moving target, so as to realize the function of high-precision measurements at sea. This article afterwards precise point positioning dynamic positioning the base station can obtain centimeter-level results, or the results of the introduction of differential positioning satellite station, in order to achieve decimeter positioning accuracy of the rover. With this dynamic measurement technique can be further improved rover measurement accuracy and reliability, save manpower, material cost of setting ground stations. In addition, GNSS dynamic positioning relative measurement technique can also be applied to UAV formation flight, maritime UAV landing, space cooperation target dynamic precision differential positioning and other fields, with some forward-looking. 


\section{Status and trends at home and abroad}

In order to obtain a better positioning accuracy at sea for coastal waters, it can be used to set up base stations on shore or islands, using differential GNSS positioning technology for high-precision Sea. By providing one or more reference stations in coastal waters along the coast can be highly accurate positioning at sea.

After years of research, the current differential GNSS positioning applications can be divided into local area differential (LADGPS, single differential base station, base station multi-differential) and wide area differential (WADGPS) according to the working principle and the difference model. The technology is based on the principle that exists between the base and rover within a certain distance of GNSS satellite observations synchronized strong correlation between the use of the rover positioning reference station to provide error correction information to improve their positioning accuracy, radius of action LADGPS Comparison small, for example, the differential radius of action is usually not more than $150 \mathrm{~km}$, then real-time positioning accuracy of subscriber stations can generally be increased to $3 \mathrm{~m} \sim 5 \mathrm{~m}$. If you use a local difference in the coastal system, and its radius is much smaller than the land.

WADGPS technical principle is to distinguish and modeling error sources GNSS observables, then the calculated source of error correction value (difference value) data communications link and transmit them to the user, in order to achieve weaken these sources of error and improve the user positioning accuracy purpose. There EGNOS ESA, mainly covering Europe; Raytheon's Wide Area Augmentation System WAAS, covering the American continent; Japan's Multi-functional Satellite Augmentation System MSAS, covering the Asian continent, in China's eastern coastal can also receive MSAS enhanced satellite signal. (Figure 1)

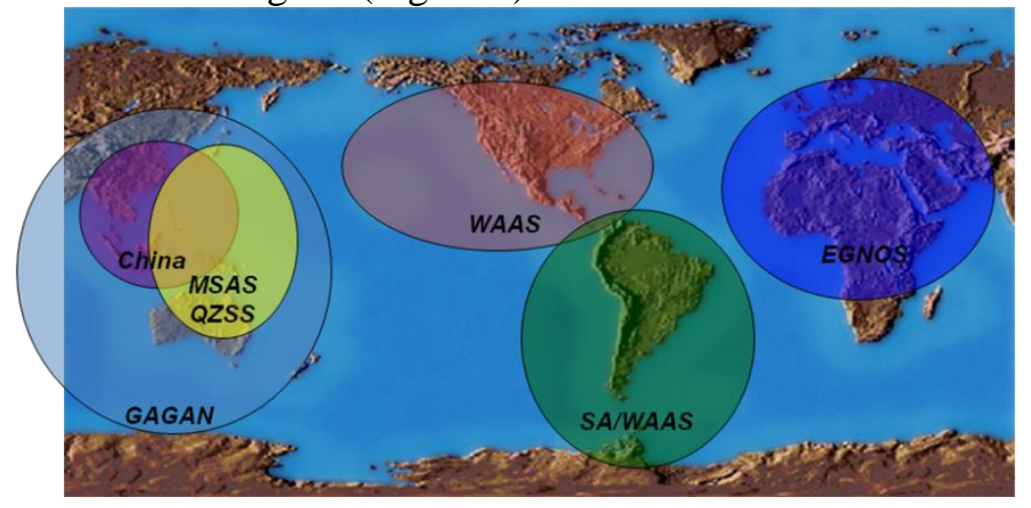

Figure 1. Global Wide Area Augmentation System Coverage

Star Station differential technique is used in place of ground satellite station broadcast on GPS differential correction signal differential technology, the technology can solve the open sea to the base station can not receive data problems, so far from the sea with high accuracy positioning becomes possible. The system uses the global distribution of 70 ground reference stations to determine the GNSS system errors, in order to achieve high accuracy real-time kinematic positioning. In the 1990s, began to study abroad to develop differential GPS satellite station, has appeared and put into use the satellite station differential GPS system is mainly: CDGPS, OmniSTAR system, SeaSTAR system, Skyfix system and StarFire system. Although the several foreign satellite-based differential GPS system is fully operational, but to enter the Chinese market, to provide users with real-time location services only Fugro's Marinestar Systems and JPL and NAVCOM company to launch the StarFire system. UniStrong company StarFire equipment and afterwards precise point positioning result comparison, in most cases the level of positioning accuracy of better than $0.3 \mathrm{~m}$, height positioning accuracy of better than $0.5 \mathrm{~m}$.

Most domestic and foreign researchers is the dynamic differential positioning reference station experiments on land or in coastal areas, offshore and no test cases far from the sea region of the analysis, there is no difference for the Beidou system dynamic accuracy and reliability is no in-depth research and analysis. The research project has some cutting-edge, with a strong guidance for subsequent precise measurements at sea. 


\section{Preliminary research programs}

a) Terrestrial base station to establish a dynamic differential positioning hardware and software environment, the formation of the sea and debug dynamic differential positioning software and hardware. Build two offshore dynamic differential measurement protocols. Option One: built on an open area of land measuring three points, the composition of the base station differential dynamic system, the three measuring three measuring points between each other can be used as baseline test accuracy. Option Two: to create two dynamic measurement points on the ship, to verify the differential positioning accuracy in a dynamic environment. In determining the relative dynamic differential positioning accuracy, based on the study of precise point positioning technology and satellite stations difference technique, relative positioning result is converted to absolute positioning results, in order to coordinate follow-up imputed.

Preliminary experiments at the reference points were admitted BDS/GPS dual frequency raw observation data, after using BDS / GPS satellite precise orbit and final clock error data, earth rotation parameters and satellite phase center corrections and other external data dynamic precise point positioning coordinates of the solution were calculation, static test results with reference solver stand in comparison with known coordinates. As shown below, the use of dual-frequency data BDS solver for $4.03 \mathrm{~cm}$ respectively integrated measurement error latitude, longitude and elevation direction, $5.46 \mathrm{~cm}$ and $3.49 \mathrm{~cm}$. Using GPS data accuracy of calculation is consistent with the BDS. (Figure 2)
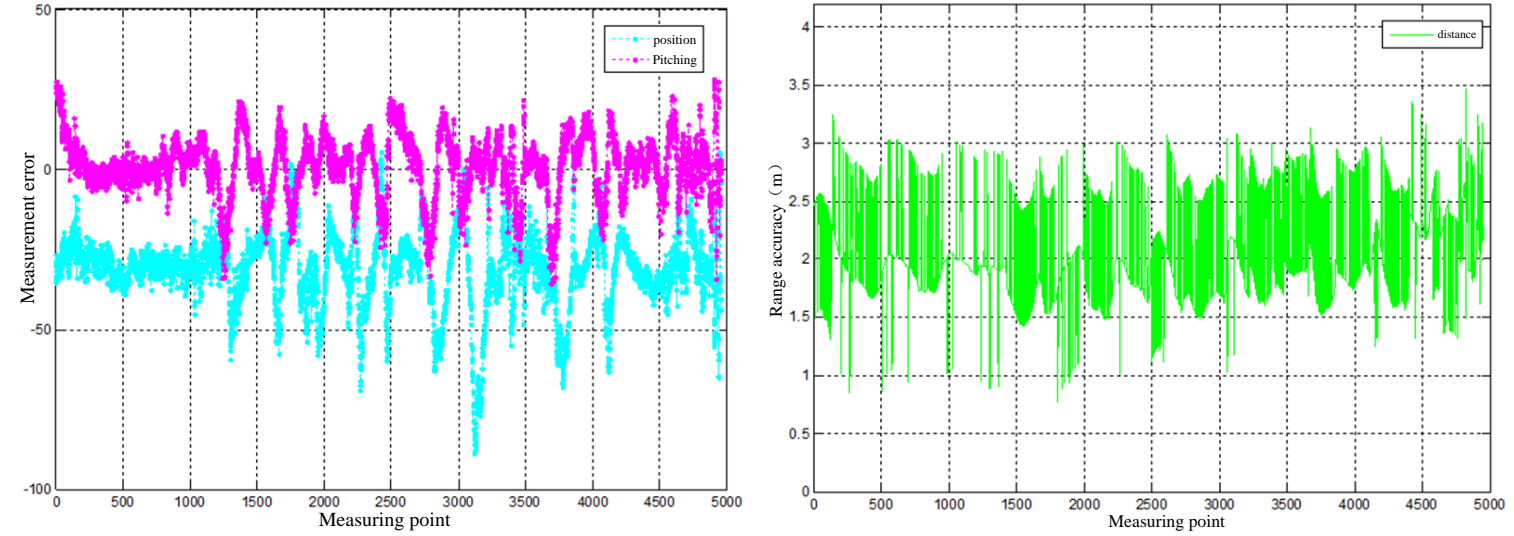

Figure 2. Fixed reference station GNSS static test

b) Study of GNSS reference station fixed and dynamic differential positioning algorithms and models, in order to use the differential positioning algorithm for land and sea access to high-precision positioning results. Mainly comprising differential positioning mode, navigation satellite frequency selection, error model selection, data processing filtering algorithm, select the navigation system, the whole cycle ambiguity, precise point positioning algorithm. By selecting different differential positioning algorithms and models, you need to find suitable land and sea data processing portfolio. BD system focuses on the choice of algorithms and models on the positioning precision of the case.

During the land, to the base station as a reference to set precision positioning system on both rovers were moving target, so he established three short baselines. In the GPS/BDS differential positioning, the main problem of double difference observation equation to be solved is to determine the integer ambiguity. If the ambiguity can be fixed to an integer to obtain a solution as a fixed solution; if the solution is a real number, the result is called a float solution. Fixed solution is generally believed that the positioning accuracy in the centimeter level positioning accuracy float solution than an order of magnitude lower fixed solution. During the sea, using a precision point positioning satellite navigation coordinates solver with an accuracy of about 1-2 meters, it is possible to complete the detection accuracy of satellite navigation. The accuracy of the detection system positioning satellite navigation positioning results with the results of comparison, the results according to the following formula statistics; (Figure 3)

$$
\Delta \xi_{i}=\xi_{i}-\xi_{0}
$$




$$
\begin{aligned}
& \sigma_{s}=\frac{1}{n} \sum_{i=1}^{n}\left(\Delta \xi_{i}\right) \\
& \sigma_{r}=\sqrt{\sum_{i=1}^{n}\left(\Delta \xi_{i}-\sigma_{s}\right)^{2} /(n-1)} \\
& \sigma_{t}=\sqrt{\sigma_{s}^{2}+\sigma_{r}^{2}}
\end{aligned}
$$

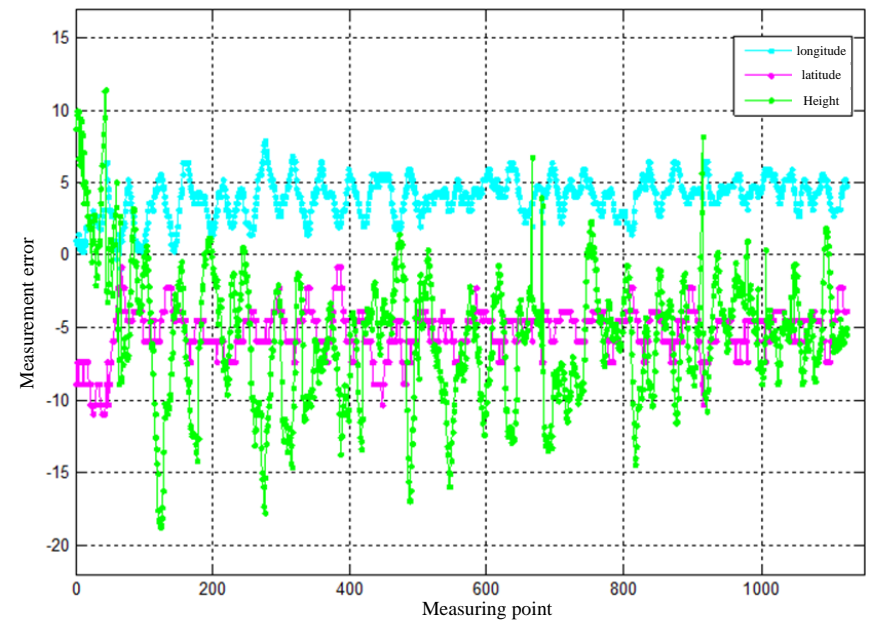

Figure 3. Differential positioning algorithm test results

c) Finally, the experimental data, analysis and evaluation of the base station differential GPS dynamic positioning accuracy, GPS and Compass systems are summarized in the test role, comb the sea to build a dynamic differential test hardware and software environment conditions.

\section{Summary}

Differential GNSS positioning technology by conducting research on the sea side of the mobile base station, the paper built GPS/BDS differential precision positioning system and its hardware and software operating environment settings to study the principles of accuracy of the detection system and the data processing step. Finally, the results of evaluation of the data processing to detect dynamic differential positioning accuracy of the results

\section{References}

[1]. Chen Jun. Summary of Application of Beidou satellite navigation and positioning system [J]. "Computer Engineering and Applications", 2015, 51 (S1): 115-118.

[2]. Wang Shijin. Location Algorithm of. GPS/BDS [J]. "Wuhan University Information Science." 2014, 39 (5): 621-625.

[3]. Wang Zhi. Modeling and experimental study of a dynamic relative positioning of the dynamic [J]. Ship Electronic Engineering 2015,248 (2): 74-76

[4]. Zhou Jian. Single-frequency GPS positioning accuracy in real-time analysis [J]. "Surveying and Mapping Science", 2015, 40 (6): 151-155. 